\title{
Peak-to-Average Power Ratio Reduction in Interleaved OFDMA Systems
}

\author{
Shamael Al-Shuhail \\ King Abdullah University \\ of Science \& Technology, \\ Thuwal, Saudi Arabia \\ Email: shamael.shuhail@kaust.edu.sa
}

\author{
Anum Ali \\ University of Texas at Austin, \\ Austin, TX 78712 USA \\ Email: anumali@utexas.edu
}

\author{
Tareq AlNaffouri \\ King Abdullah University \\ of Science \& Technology, \\ Thuwal, Saudi Arabia \\ Email: tareq.alnaffouri@kaust.edu.sa
}

\begin{abstract}
Orthogonal frequency division multiple access (OFDMA) systems suffer from several impairments, and communication system engineers use powerful signal processing tools to combat these impairments and to keep up with the capacity/rate demands. One of these impairments is high peak-to-average power ratio (PAPR) and clipping is the simplest peak reduction scheme. However, in general, when multiple users are subjected to clipping, frequency domain clipping distortions spread over the spectrum of all users. This results in compromised performance and hence clipping distortions need to be mitigated at the receiver. Mitigating these distortions in multiuser case is not simple and requires complex clipping mitigation procedures at the receiver. However, it was observed that interleaved OFDMA presents a special structure that results in only self-inflicted clipping distortions (i.e., the distortions of a particular user do not interfere with other users). In this work, we prove analytically that distortions do not spread over multiple users (while utilizing interleaved carrier assignment in OFDMA) and construct a compressed sensing system that utilizes the sparsity of the clipping distortions and recovers it on each user. We provide numerical results that validate our analysis and show promising performance for the proposed clipping recovery scheme.
\end{abstract}

Index Terms-Interleaved OFDMA, PAPR Reduction, Compressed Sensing, Nonlinear Distortions.

\section{INTRODUCTION}

OFDMA is a widely used modulation technique adopted by many wired and wireless technologies. It is a multicarrier modulation technique based on modulating a number of orthogonal carriers [1]. OFDMA allows access to multiple users and assigns a subset of available subcarriers to each user. Because of the orthogonal nature of the subcarriers, there is no inter-user interference [1]. OFDMA systems offer a considerably high spectral efficiency, tolerance against multipath delay spread, immunity to the frequency selective fading and power efficiency [2], [3]. On the other hand, high PAPR is the main disadvantage of OFDMA signals. When high PAPR signals in mobile communications uplink are subjected to a power amplifier, the signal is distorted [1].

Many techniques have been proposed to reduce the PAPR. Some examples are phase optimization [4], nonlinear companding transforms [5], Tone Reservation and Tone injection $[6,7]$. One of the simplest approaches used for PAPR reduction is clipping at the transmitter and recovering the clipping signal using a recovery technique at the receiver side. The clipping signal is observed to be sparse by nature i.e., non-zero elements only at a relatively small number of locations in the signal. Due to the sparsity of the clipping signal, Compressed Sensing (CS) techniques have been used for PAPR reduction e.g., [8-10].

Unlike clipping in the single user scenario, when multiusers are clipped at their respective transmitters, the clipping distortions from all users overlap in the frequency domain [11]. As the measurements of CS based clipping recovery are taken in frequency domain, this distortion overlap has a dire impact on the reliability of the measurements. However, it is observed that interleaved OFDMA (i.e., OFDMA setup where the subcarrier allocation is interleaved), clipping distortions are only self-inflicted, i.e., the clipping distortions from user $u$ are confined only to the subcarriers that are allocated to the user $u$ itself [12]. This, however, is not true for consecutive carrier allocation, where distortion overlap is a serious issue from CS based clipping recovery viewpoint [11].

In this paper, we present a mathematical proof authenticating the observation that clipping distortions are self-inflicted for interleaved OFDMA. We start by introducing the data model for a clipped OFDMA system in section II. Then in section III we explain the proposed solution to clipping distortion mitigation. We proceed in section III by providing a mathematical proof of the solution. We then present the CS recovery model in section IV. Moreover, we illustrate the simulation results in section V. Finally, section VI concludes the paper.

\section{DATA MODEL}

In an OFDMA system, incoming bits are divided into $N$ low rate parallel streams. An $\mathrm{N}$ dimensional data vector $\mathbf{X}=$ $\left[X^{u}(0), X^{u}(1), \ldots, X^{u}(N-1)\right]^{\top}$ results after mapping the $N$ parallel streams to an $M$-ary QAM and the $U$ notation defines the user that the subcarrier is allocated to $(u=0,1, \ldots U-$ $1)$. We find the time domain signal vector $\mathbf{x}$ by applying an $N$ dimensional IDFT $\mathbf{F}^{H}$, so that $\mathbf{x}=\mathbf{F}^{H} \mathbf{X}$. Hence, the resulting complex packet of the transmitted OFDM signals can be expressed as the following: 
$x^{u}(n)=\frac{1}{\sqrt{N}} \sum_{k=0}^{N-1} X^{u}(k) \exp \left(j \frac{2 \pi k n}{N}\right) \quad 0 \leq n \leq N-1$

where $x^{u}(n)$ is the $n^{\text {th }}$ element of the transmitted signal $\mathbf{x}$ for user $u$.

The transmitted signal's PAPR can be expressed as following:

$$
\text { PAPR }=\frac{\max _{0 \leq n \leq N-1}\left|x^{u}(n)\right|^{2}}{\mathrm{E}\left\{\left|x^{u}(n)\right|^{2}\right\}}
$$

In a $U$ user OFDMA system, each user is assigned a total of $P$ sub-carriers (where $P=N / U$ ) and each sub-carrier is assigned to one user only. The $P$ dimensional data vector $\mathbf{X}^{u}$ is then mapped to the allocated subcarriers using a resource allocation matrix $\mathbf{M}^{u}$ of size $N \times P$. We can define $\mathbf{M}^{u}$ as a partial identity matrix of size $N \times P$ with the selected columns being the corresponding locations of the subcarriers designated to user $u$. Now the time domain data from user $u$ can be written as

$$
\mathbf{x}^{u}=\mathbf{F}^{H} \mathbf{M}^{u} \mathbf{X}^{u}
$$

where $\mathbf{M}^{u} \mathbf{X}^{u}$ is the $N$ dimensional data vector with nonzero values only at locations of the subcarriers allocated for the $u^{\text {th }}$ user. To reduce the PAPR in $\mathbf{x}^{u}$, the signal is subjected to clipping. The clipping operation limits the maximum amplitude to the threshold $\gamma^{u}$ [13]. The clipping operation can be mathematically written as [11]:

$$
x_{c}^{u}(n)= \begin{cases}\gamma^{u} e^{j \angle x^{u}(n)} & \text { if }\left|x^{u}(n)\right|>\gamma^{u} \\ x^{u}(n) & \text { otherwise }\end{cases}
$$

where $x_{c}^{u}(n)$ is the $n^{\text {th }}$ element of the $u^{\text {th }}$ signal $\mathbf{x}^{u}$ after clipping, and $\angle x^{u}(n)$ is the phase of the original signal $x^{u}(n)$. The clipping threshold $\gamma^{u}$ is related to the clipping ratio (CR) as $\mathrm{CR}^{u}=\gamma^{u} / \sigma^{u}$ [11], where $\sigma^{u}$ is the root-mean-squared power of the OFDM signal transmitted by the $u^{\text {th }}$ user. This results in the following expression:

$$
\mathbf{x}_{c}^{u}=\mathbf{x}^{u}+\mathbf{c}^{u}
$$

where $\mathbf{c}^{u}$ is the clipping signal from the $u^{\text {th }}$ user.

The clipped signal $\mathbf{x}_{c}^{u}$ is then transmitted through a channel of length $N_{c}$ with impulse response $\mathbf{h}^{u}=$ $\left[h^{u}(0), h^{u}(1), \ldots, h^{u}\left(N_{c}-1\right)\right]^{\top}$. Hence, the received time domain signal can be expressed as $\mathbf{y}^{u}=\mathbf{H}^{u} \mathbf{x}_{c}^{u}$, where $\mathbf{H}^{u}$ is the circulant channel matrix which can be diagonalized using the DFT matrix $\mathbf{F}_{N \times N}$ and can be expressed as $\mathbf{H}^{u}=\mathbf{F}^{H} \mathbf{D}^{u} \mathbf{F}$. Where $\mathbf{D}^{u}$ is a diagonal matrix with the partial Fourier transform of the impulse response of the $u^{\text {th }}$ user i.e., $\overline{\mathbf{F}} \mathbf{h}^{u}$ on its diagonal. The received time domain signal at the base station can be written as

$$
\mathbf{y}=\sum_{u=0}^{U-1} \mathbf{H}^{u}\left(\mathbf{x}^{u}+\mathbf{c}^{u}\right)+\mathbf{z}
$$

where $\mathbf{y}$ is the combined channel output of all users plus additive white Gaussian noise (AWGN), $\mathbf{z} \sim \mathcal{C N}\left(0, \sigma_{z}^{2} \mathbf{I}_{N}\right)$.

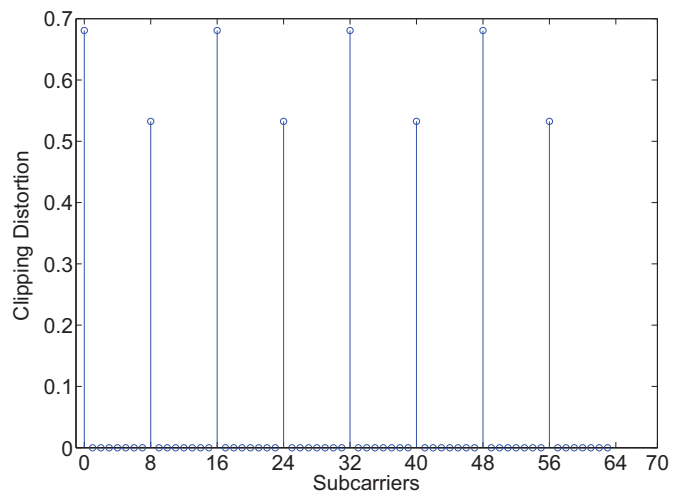

Fig. 1. The clipping distortions of the $0^{\text {th }}$ user.

The frequency domain received signal can be obtained by the DFT operation as following

$$
\mathbf{Y}=\sum_{u=0}^{U-1} \mathbf{D}^{u} \mathbf{M}^{u} \mathbf{X}^{u}+\sum_{u=0}^{U-1} \mathbf{D}^{u} \mathbf{M}^{u} \mathbf{C}^{u}+\mathbf{Z}
$$

where the frequency domain white Gaussian vector $\mathbf{Z}$ has the same statistics as $\mathbf{z}$. The column vector resulting from $\mathbf{D}^{u} \mathbf{M}^{u} \mathbf{X}^{u}$ at each user $u$ will have non-zero values only at locations corresponding to the sub-carriers allocated to the $u^{\text {th }}$ user.

\section{Special CASE: Interleaved OFDMA}

With interleaved allocation, subcarriers are allocated to each user in an alternating manner. In the case of an OFDMA signal with $N$ subcarriers, $U$ users, and $P=N / U$ subcarriers per user, the $(k, l)^{\text {th }}$ element of the resource allocation matrix $\mathbf{M}^{u}$ is given by

$$
m^{u}(k, l)= \begin{cases}1, & k=u+U l, \quad 0 \leq l \leq P-1 \\ 0, & \text { otherwise }\end{cases}
$$

At this stage, we conjecture that for the interleaved subcarrier assignment the clipping distortion is only self-inflected, i.e., when the clipping distortions from the user $u$ are viewed in frequency domain, they only fall on the sub-carriers allocated to user $u$. The clipping vector in the frequency domain i.e., $\mathbf{M}^{u} \mathbf{C}^{u}$ looks like

$$
\mathbf{M}^{u} \mathbf{C}^{u}=\left[\begin{array}{lllllll}
\mathbf{0}_{u} & C^{u}(0) & \mathbf{0}_{U-1} & C^{u}(1) & \ldots & C^{u}(P-1) & \mathbf{0}_{U-(u+1)}
\end{array}\right]_{(9)}^{\top}
$$

where $\mathbf{0}_{u}$ is the zero vector of length $u$ and $C^{u}(p)$ is the $p^{\text {th }}$ element of the $u^{\text {th }}$ user in the clipping signal. Figure 1 illustrates the clipping signal of the $0^{\text {th }}$ user when $N=64$ and the overall number of users is $U=8$. As observed, the clipping distortion only occurs at the subcarriers allocated for user 0 .

In the following subsection we outline the mathematical proof that shows that (9) is always true. 


\section{A. Proof of Self-Inflected Clipping Distortion in Interleaved OFDMA}

To start the proof, we first show the data generated and allocated for user $u$. For simplicity we will show when the $0^{\text {th }}$ user is subjected to clipping i.e., $u=0$, the allocated data will look like the following

$\mathbf{M}^{0} \mathbf{X}^{0}=\left[\begin{array}{llllll}f^{0}(0) & \mathbf{0}_{U-1} & f^{0}(1) & \ldots & f^{0}(P-1) & \mathbf{0}_{U-1}\end{array}\right]^{\top}$

where $f^{0}(p)$ is the $p^{\text {th }}$ element of the $0^{\text {th }}$ user's data in the frequency domain.

Since data is clipped in the time domain, the result from (10) is multipied by the IDFT matrix $\mathbf{F}^{H}$ as following

$$
\begin{aligned}
& \mathbf{F}^{H}\left[\begin{array}{llllll}
f^{0}(0) & \mathbf{0}_{U-1} & f^{0}(1) & \ldots & f^{0}(P-1) & \mathbf{0}_{U-1}
\end{array}\right]^{\top} \\
& =\frac{1}{\sqrt{N}}\left[\begin{array}{c}
f^{0}(0) e^{j \frac{2 \pi}{N}(0)(0)}+\ldots+f^{0}(P-1) e^{j \frac{2 \pi}{N}(0)(P-1)(U)} \\
f^{0}(0) e^{j \frac{2 \pi}{N}(1)(0)}+\ldots+f^{0}(P-1) e^{j \frac{2 \pi}{N}(1)(P-1)(U)} \\
\vdots \\
f^{0}(0) e^{j \frac{2 \pi}{N}(N-1)(0)}+\ldots+f^{0}(P-1) e^{j \frac{2 \pi}{N}(N-1)(P-1)(U)}
\end{array}\right] \\
& =\left[\begin{array}{c}
t^{0}(0) \\
t^{0}(1) \\
\vdots \\
t^{0}(N-1)
\end{array}\right]
\end{aligned}
$$

where $t^{0}(n)$ is the $n^{\text {th }}$ element of the $0^{\text {th }}$ user's data in the time domain. It is noted that the time domain signal found in (11) is periodic with period $P$, i.e., $N / U$. To prove that, let us show that $t^{0}(r)=t^{0}(r+P)$ where $r$ could be any row that satisfies $0 \leq r \leq P-1$. The proof proceeds as following

$$
\begin{gathered}
\frac{1}{\sqrt{N}}\left(f^{0}(0) e^{j \frac{2 \pi}{N}(r)(0)}+\ldots+f^{0}(P-1) e^{j \frac{2 \pi}{N}(r)(P-1)(U)}\right) \\
\Uparrow \\
\frac{1}{\sqrt{N}}\left(f^{0}(0) e^{j \frac{2 \pi}{N}(r+P)(0)}+\ldots+f^{0}(P-1) e^{j \frac{2 \pi}{N}(r+P)(P-1)(U)}\right)
\end{gathered}
$$

Let us deal with the problem term wise and show that the first terms of (12) and (13) are equal. To establish that, we simplify the terms even more, yielding

$$
f^{0}(0) e^{j \frac{2 \pi}{N}(r)(0)}=f^{0}(0) \times 1=f^{0}(0)
$$

from (12) and

$$
f^{0}(0) e^{j \frac{2 \pi}{N}(r+P)(0)}=f^{0}(0) \times 1=f^{0}(0)
$$

from (13). Hence, from (14) and (15) we see that the terms are indeed equal.

We proceed by proving the second terms of (12) and (13) are equal as well. This will result in the following (note that $U=N / P$ and $\left(e^{j 2 \pi}\right)^{K}=1$ where $K$ is an integer)

$$
f^{0}(1) e^{j \frac{2 \pi}{N}(r)(U)}
$$

from (12) and

$$
\begin{aligned}
& f^{0}(1) e^{j \frac{2 \pi}{N}(r+P)(U)}=f^{0}(1) \times e^{j \frac{2 \pi}{N}(r U+P U)} \\
& =f^{0}(1) \times e^{j \frac{2 \pi}{N}(r U)} \times e^{j \frac{2 \pi}{N}(P U)} \\
& =f^{0}(1) \times e^{j \frac{2 \pi}{N}(r U)} \times e^{j \frac{2 \pi}{N}\left(P \frac{N}{P}\right)}=f^{0}(1) \times e^{j \frac{2 \pi}{N}(r U)} \times e^{j 2 \pi} \\
& =f^{0}(1) \times e^{j \frac{2 \pi}{N}(r U)} \times 1=f^{0}(1) e^{j \frac{2 \pi}{N}(r U)}
\end{aligned}
$$

from (13). Hence, from (16) and (17) we see that the second terms are also equal.

The process of proving the equality of the terms goes on until we reach the last terms. Here, we show the proof of equality for the last term of (12) and (13),

$$
f^{0}(P-1) e^{j \frac{2 \pi}{N}(r)(P-1)(U)}
$$

from (12) and

$$
\begin{aligned}
& f^{0}(P-1) e^{j \frac{2 \pi}{N}(r+P)(P-1)(U)} \\
& =f^{0}(P-1) \times e^{j \frac{2 \pi}{N}(r)(P-1)(U)} \times e^{j \frac{2 \pi}{N}(P)(P-1)\left(\frac{N}{P}\right)} \\
& =f^{0}(P-1) \times e^{j \frac{2 \pi}{N}(r)(P-1)(U)} \times\left(e^{j 2 \pi}\right)^{(P-1)} \\
& =f^{0}(P-1) \times e^{j \frac{2 \pi}{N}(r)(P-1)(U)} \times 1 \\
& =f^{0}(P-1) e^{j \frac{2 \pi}{N}(r)(P-1)(U)}
\end{aligned}
$$

from (13). Hence, from (18) and (19) we see that the final terms are also equal.

As a result, the time domain signal from (11) for any user is indeed periodic for period $P$. The signal in (11) now goes through the clipping process leading to a clipping signal $\mathbf{c}^{u}$ that is periodic for period $P$ as well, (note that for consistency purposes we keep operating with the $0^{\text {th }}$ user, however the same approach may work for any user $u$ )

$$
\mathbf{c}^{0}=\left[\begin{array}{c}
c^{0}(0) \\
c^{0}(1) \\
\vdots \\
c^{0}(N-1)
\end{array}\right]
$$

To prove the self-inflicted clipping distortion of the $0^{\text {th }}$ user, we transform (20) to frequency domain using the DFT matrix F. A property of the Fourier matrix, the reciprocity theorem, will result in a signal that restores the format of (10) (non-zero values only at locations of the harmonics of $U$ i.e., $0, \pm U, \pm 2 U, \ldots$, otherwise the value is zero). Hence, the resulting frequency domain clipping signal for the $0^{\text {th }}$ user will look as following

$$
\mathbf{M}^{0} \mathbf{C}^{0}=\left[\begin{array}{llllll}
C^{0}(0) & \mathbf{0}_{U-1} & C^{0}(1) & \ldots & C^{0}(P-1) & \mathbf{0}_{U-1}
\end{array}\right]^{\top}
$$

A general way to write (21) for any user $u$ clipped is as in (9). 


\section{Compressive Sensing Recovery Model}

Now that we have proved that the clipping distortions from different users do not spread or overlap between different users, we seek to recover the clipping signal $\mathbf{M}^{u} \mathbf{C}^{u}$ at the receiver's side. To do that we show the recovery model used to reconstruct the original signal $\mathbf{M}^{u} \mathbf{X}^{u}$. Knowing that the data is allocated in an interleaved manner, the CS model can be constructed from (7). Taking into account that the recovery process works with each user individually in (7) i.e., selecting the entries in $\mathbf{Y}$ and $\mathbf{Z}$ that correspond to the subcarriers allocated to user $u$, resulting in the following

$$
\mathbf{Y}^{u}=\mathbf{D}^{u} \mathbf{M}^{u} \mathbf{X}^{u}+\mathbf{D}^{u} \mathbf{M}^{u} \mathbf{C}^{u}+\mathbf{Z}^{u}
$$

We further extend (22) to an equation with sparse unknown we start with $\mathbf{Y}^{u}-\mathbf{D}^{u} \mathbf{M}^{u} \mathbf{X}^{u}=\mathbf{D}^{u} \mathbf{M}^{u} \mathbf{C}^{u}+\mathbf{Z}^{u}$. By reserving some carriers to sense clipping from user $u$, we are able to get the measurement vector for the model. The number of reserved tones is said to be $s$. The same goes to $\mathbf{D}^{u}$, the matrix will only have the rows corresponding to those reserved tones, changing the size of $\mathbf{D}^{u}$ to be $s \times N$. Hence, we get

$$
\mathbf{Y}_{s}^{u}=\mathbf{D}_{s}^{u} \mathbf{M}^{u} \mathbf{C}^{u}+\mathbf{Z}_{s}^{u}
$$

where $\mathbf{Y}_{s}^{u}$ and $\mathbf{Z}_{s}^{u}$ are of size $s \times 1$.

Since our sparse unknown is $\mathbf{c}^{u}$, we can transform $\mathbf{M}^{u} \mathbf{C}^{u}$ to $\mathbf{c}^{u}$ by using the DFT matrix as $\mathbf{M}^{u} \mathbf{C}^{u}=\mathbf{F} \mathbf{c}^{u}$. This results in the following

$$
\mathbf{Y}_{s}^{u}=\mathbf{D}_{s}^{u} \mathbf{F} \mathbf{c}^{u}+\mathbf{Z}^{u}
$$

where $\mathbf{Y}_{s}^{u}, \mathbf{D}_{s}^{u} \mathbf{F}$, and $\mathbf{c}^{u}$ are the measurement vector, the sensing matrix, and the unknown sparse signal respectively.

\section{Simulation Results}

An OFDMA system with $N=128$ subcarriers is simulated, with $U=2$ active users accessing the frequency resources. The data is modulated using a QAM constellation of order $Q=64$. The channel length is $N_{c}=\frac{N}{4}$.

As observed from figure 2, the fast Bayesian matching pursuit (FBMP) [14] is successfully recovering the original signal back due to the absence of clipping distortions spreading from different users. The CR selected in figure 2 is $\sqrt{3}$ and the number of reserved tones used is $s=32$.

In figure 3, we see the result of FBMP recovery when subcarriers are assigned in a non-interleaving manner, i.e., consecutive assignment. As observed, the recovery performance is very poor due to overlapping clipping distortions from both users.

\section{CONCLUSION}

In this work, we addressed the problem of PAPR reduction in interleaved OFDMA system. It was observed that due to the time domain periodicity of the clipped signal from each user, the combined OFDMA signal at the receiver is separable in frequency domain. Hence, we formed a subsystem for each user and applied compressed sensing to recover the

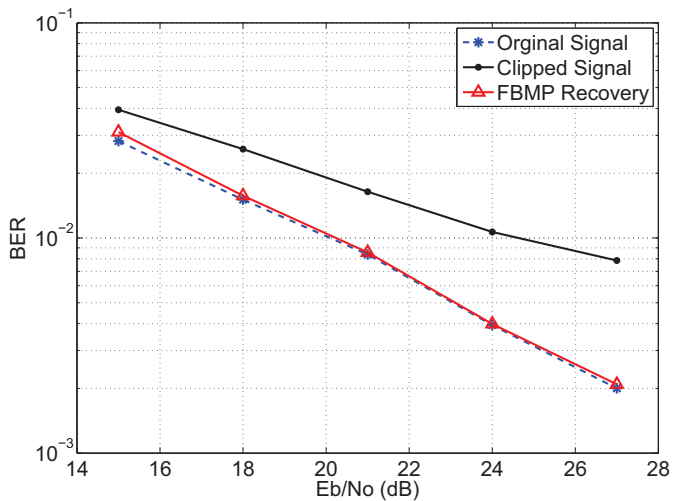

Fig. 2. BER performance of the FBMP recovery scheme as a function of $E_{b} / N_{0}$ (interleaved carrier assignment).

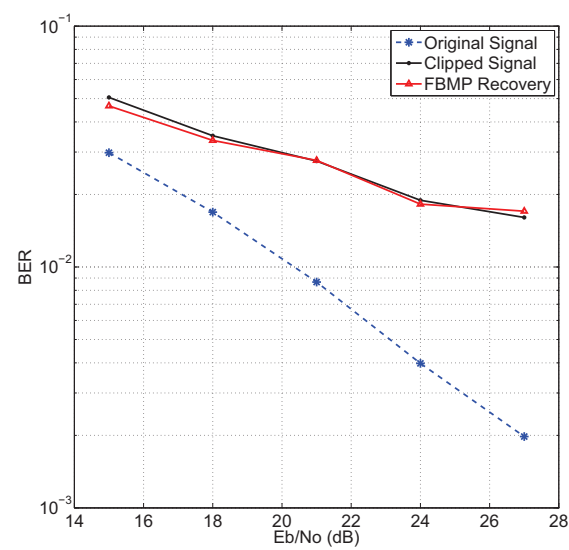

Fig. 3. BER performance of the FBMP recovery scheme as a function of $E_{b} / N_{0}$ (consecutive carrier assignment).

clipped signal. Simulation results indicate that following such an approach can give bit error rate performance as good as unclipped signal, albeit at the expense of rate loss incurred by the reserved tones.

\section{REFERENCES}

[1] D. Sinanovic, G. Sisul, and B. Modlic, "Comparison of BER characteristics of OFDM and SC-FDMA in frequency selective channels," in Systems, Signals and Image Processing (IWSSIP), 2011 18th International Conference on, vol., no., pp.1,4, 16-18 June 2011.

[2] Y.Wu and W. Y. Zou, "Orthogonal frequency division multiplexing: A multi-carrier modulation scheme," in IEEE Trans. Consumer Electronics, vol. 41, no. 3, pp. 392399, Aug 1995

[3] W. Y. Zou and Y. Wu, "COFDM: An overview," IEEE Trans. Broadcasting, vol. 41, no. 1, pp. 18, Mar 1995.

[4] V. Tarokh and H. Jafarkhani, "On the computation and reduction of the peak-to-average power ratio in multicarrier communications," IEEE Trans. Commun., vol. 48, no. 1, pp. 3744, Jan 2000.

[5] X. B. Wang, T. T. Tjhung, and C. S. Ng, "Reduction of peak-to-average power ratio of OFDM system using A companding technique," IEEE Trans. Broadcast., vol. 45, no. 3, pp. 303-307, 1999. 
[6] J. Tellado, "Peak to Average Power Ratio Reduction for Multicarrier Modulation," PhD thesis, University of Stanford, Stanford, 1999.

[7] S. S. Yoo, S. Yoon, S. Y. Kim, and I. Song, "A novel PAPR reduction scheme for OFDM systems: Selective mapping of partial tones (SMOPT)," IEEE Trans. Consumer Electronics, vol. 52, no. 1, pp. 4043, 2006.

[8] E. B. Al-Safadi and T. Y. Al-Naffouri, "Peak reduction and clipping mitigation in OFDM by augmented compressive sensing," IEEE Trans. Signal Process., vol. 60, no. 7, pp. 3834 - 3839, July 2012.

[9] A. Al-Rabah, M. Masood, A. Ali, and T. Y. Al-Naffouri, "Receiverbased Bayesian PAPR reduction in OFDM," in Signal Process. Conf. (EUSIPCO), 2013 Proc. of the 21st European, vol., no., pp. 1-5, 9-13 Sept 2013.

[10] A. Ali, A. Al-Rabah, M. Masood, and T. Y. Al-Naffouri, "Receiver-based recovery of clipped OFDM signals for PAPR reduction: A Bayesian approach," in Access, IEEE, vol. 2, no., pp.1213 - 1224, 2014.

[11] A. Ali, A. Al-Zahrani, T. Y. Al-Naffouri, and A. Naguib, "Receiver Based PAPR Reduction in OFDMA," in ICASSP, 2014 IEEE Int. Conf., vol., no., pp. 8087-8091, 4-9 May.

[12] Z. Cao, H. van den Boom, E. Tangdiongga, and T. Koonen, "Interleaved and partial transmission interleaved optical coherent orthogonal frequency division multiplexing," in Optical Soc. of Amer, vol. 39, no. 7, pp. 2179-2182, 2014.

[13] R. Gross and D. Veeneman, "SNR and spectral properties for a clipped DMT ADSL signal," in IEEE Intl. Conf. Commun., vol., no., pp.843-847, vol.2, 1994.

[14] P. Schniter, L. C. Potter, and J. Ziniel, "Fast Bayesian matching pursuit," in Proc. Inform. Theory \& Appl. Workshop, Feb 2008. 